9. F. D. Murnaghan, The theory of group representations, Baltimore, 1938.

10. G. P6lya, Kombinatorische Anzahlbestimmungen fïr Gruppen, Graphen und chemische Verbindungen, Acta Math. vol. 68 (1937) pp. 145-254.

11. R. J. Riddell, Contributions to the theory of condensation, University of Michigan Dissertation, 1951.

UNIVERSITY OF MichigaN

\title{
TWO NOTES ON RECURSIVELY ENUMERABLE SETS
}

\section{J. C. E. DEKKER}

Introduction. These notes are based on E. L. Post's paper Recursively enumerable sets of positive integers and their decision problems ${ }^{1}$ to which we shall refer as RES. The reader is assumed to be familiar with $\$ \S 1-5$ and 9 of this paper. In the first note we shall discuss some algebraic properties of simple and hypersimple sets. In the second note we shall prove the existence of a recursively enumerable set which is neither recursive nor creative nor simple and discuss its degree of unsolvability relative to one-one reducibility and relative to many-one reducibility.

Notations and terminology. A collection of non-negative integers is called a set, a collection of sets is called a class. An empty collection is considered as a special case of a finite collection. Non-negative integers and functions are denoted by small Latin letters, sets by small Greek letters, and classes by capital Latin letters. The Boolean operations are denoted by " + " for addition, " $X$," "." or juxtaposition for multiplication, " sion. Proper inclusion between classes is denoted by " $\mathrm{C}_{+}$."

$\epsilon={ }_{d f}$ the set of all non-negative integers.

$0={ }_{d f}$ the empty set.

$\kappa={ }_{d f}$ the complete set defined on p. 295 of RES.

$\zeta={ }_{d f}$ the simple set defined on p. 298 of RES.

$P={ }_{d f}$ the class of all sets whose complement is finite.

$Q={ }_{d f}$ the class of all finite sets.

$E={ }_{d f}$ the class of all recursive sets.

$D={ }_{d j} E-(P+Q)$.

Presented to the Society, November 29, 1952; received by the editors October 15, 1952.

${ }^{1}$ Bull. Amer. Math. Soc. vol. 50 (1944) pp. 284-316. 
$F={ }_{d f}$ the class of all recursively enumerable (r.e.) sets.

$Z={ }_{d f}$ the class of all simple sets.

$Z_{0}={ }_{d f}$ the class of all hypersimple sets.

\section{Note 1. SOME PROPERTIES OF SIMPLE SETS}

1. Preliminaries. A set is called immune if it is infinite, but has no infinite r.e. subset; a set $\alpha$ is called simple if $\alpha$ is r.e. and $\alpha^{\prime}$ immune. Clearly $Z C_{+} F-E$. The function $f(n)$ is called a recursive permutation if it is a recursive function which maps $\epsilon 1-1$ on itself. The sets $\alpha$ and $\beta$ are called isomorphic (notation: $\alpha \cong \beta$ ) if there exists a recursive permutation which maps $\alpha$ on $\beta$. The class $S$ is called recursively closed if it contains with any set $\alpha$ also all sets which are isomorphic with $\alpha$. The classes $P, Q, E, D, F, Z, Z_{0}$ are obviously recursively closed. Suppose $L$ is a class of sets which is a lattice relative to + and $X$. The subclass $S$ of $L$ is called a dual ideal in $L$ if : (1) $S$ is closed under $\times$, (2) if $\alpha \in S$ and $\beta \in L$, then $\alpha+\beta \in S$. It is easily verified that the second condition may be replaced by (2*) if $\alpha \in S, \beta \in L$, and $\alpha \subset \beta$, then $\beta \in S$. Observe that $E$ is a Boolean algebra, while $F$ is a distributive lattice with a null element (namely 0 ) and a one element (namely $\boldsymbol{\epsilon}$.

Theorem 1.1. Any two sets in $D$ are isomorphic.

Proof. If $\gamma, \delta \in D$ there exist $1-1$ recursive functions $c(n), c^{\prime}(n)$, $d(n), d^{\prime}(n)$ which range over $\gamma, \gamma^{\prime}, \delta, \delta^{\prime}$ respectively. Let $f(c(n))$ $={ }_{d f} d(n)$ and $f\left(c^{\prime}(n)\right)={ }_{d f} d^{\prime}(n)$, then it is easily verified that $f(n)$ is a recursive permutation which maps $\gamma$ on $\delta$.

Theorem 1.2. If $\sigma \in F-Q$ and $\delta \in D$, we can find a set $\tau$ such that $\delta \subset \tau$ and $\tau \cong \sigma$.

Proof. We can effectively find a set $\gamma \in D$ which is a subset of $\sigma$. By the preceding theorem there exists a recursive permutation which maps $\gamma$ on $\delta$, say $f(n)$. Let $\tau=f(\sigma)$, then $\tau \cong \sigma$; moreover $\gamma \subset \sigma$ implies $f(\gamma) \subset f(\sigma)$, i.e. $\delta \subset \tau$.

Theorem 1.3. The r.e. set $\sigma$ is simple if and only if $\sigma^{\prime} \notin Q$ and $\sigma \cdot \alpha \notin Q$ for every $\alpha \in F-Q$.

Proof. We can restrict our attention to the "only if" part, since the "if" part is obvious. If $\sigma$ is simple, then $\sigma^{\prime}$ is infinite, because $\sigma^{\prime}$ is immune. Moreover $\sigma \cdot \alpha=0$ for $\alpha \in F-Q$ is impossible, since it would imply $\sigma^{\prime} \supset \alpha$, while $\sigma^{\prime}$ is immune. But if $\sigma$ had only finitely many elements in common with the set $\alpha \in F-Q, \sigma$ would have no element in common with the set $\alpha-\sigma \cdot \alpha \in F-Q$. Thus $\sigma \cdot \alpha \notin Q$ for every 
$\alpha \in F-Q$.

The infinite sequence $\left\{\alpha_{n}\right\}$ of nonempty, finite sets is called strictly r.e. if there exist recursive functions $a(m, n)$ and $b(n)$ such that for every $n, \alpha_{n}=\{a(n, 0), \cdots, a(n, b(n))\}$. A strictly r.e. infinite sequence $\left\{\alpha_{n}\right\}$ of nonempty finite sets is called an array, the elements $\alpha_{n}$ of $\left\{\alpha_{n}\right\}$ are called the rows of the array. The array $\left\{\alpha_{n}\right\}$ is called discrete if $\alpha_{m}$ and $\alpha_{n}$ are disjoint for $m \neq n$. We say that the set $\alpha$ includes the $i$ th row of the array $\left\{\beta_{n}\right\}$ if $\alpha \supset \beta_{i}$. The set $\alpha$ is called hypersimple if $\alpha$ is r.e., $\alpha^{\prime}$ infinite, and $\alpha$ includes at least one row of every discrete array. Every hypersimple set is simple, since the recursive function $b(n)$ mentioned above may be identically 0 . Post proved the existence of a hypersimple set [RES pp. 305-308] and the existence of a simple set which is not hypersimple [RES p. 298]. Hence $Z_{0} C_{+} Z$.

THEOREM 1.4. The r.e. set $\sigma$ is hypersimple if and only if $\sigma^{\prime} \notin Q$ and $\sigma$ includes infinitely many rows of every discrete array.

Proof. We can restrict our attention to the "only if" part, the "if" part being obvious. Let $\sigma$ be hypersimple; then $\sigma^{\prime} \notin Q$ since $\sigma$ is simple. Suppose $\sigma$ included only finitely many rows of the discrete array $\left\{\alpha_{n}\right\}$. Let $r$ be the greatest number $n$ such that $\sigma \supset \alpha_{n}$. Then $\sigma$ would include no row of the discrete array $\left\{\alpha_{(r+1)+n}\right\}$; this would contradict the fact that $\sigma$ is hypersimple.

2. The main result. We can now prove some algebraic properties of simple and hypersimple sets.

TheOREM 1.5. (1) The product of two simple sets is simple.

(2) The sum of two simple sets is either simple or belongs to $P$.

(3) There exist two simple sets whose sum equals $\epsilon$.

(4) $Z+\ddot{P}$ is a dual ideal in the lattice $F$.

Proof. (1) Let $\alpha, \beta \in Z$. By Theorem 1.3 it is sufficient to prove that $(\alpha \beta)^{\prime} \notin Q$ and that $\alpha \beta \cdot \gamma \notin Q$ for every $\gamma \in F-Q$. Clearly $(\alpha \beta)^{\prime}$ is infinite, since $\alpha^{\prime}$ is infinite. Suppose $\gamma \in F-Q$; then $\beta \gamma \in F-Q$ because $\beta \in Z$, and $\alpha \beta \cdot \gamma=\alpha \cdot \beta \gamma \in F-Q$ because $\alpha \in Z$ and $\beta \gamma \in F-Q$.

(2) Let $\alpha, \beta \in Z$. Either $\alpha+\beta \in P$ or $\alpha+\beta \notin P$. In the latter case $\alpha+\beta \in Z$, since $\alpha \subset \alpha+\beta$ and $\alpha \in Z$.

(3) Let $\delta \in D, \sigma \in Z$. Then $\delta^{\prime} \in D$ and by Theorem 1.2 there exist sets $\alpha$ and $\beta$ such that $\delta \subset \alpha, \delta^{\prime} \subset \beta, \alpha \cong \sigma, \beta \cong \sigma$. Then $\alpha$ and $\beta$ are simple, since $Z$ is recursively closed; moreover $\delta+\delta^{\prime} \subset \alpha+\beta$, hence $\alpha+\beta=\epsilon$.

(4) The product of two sets in $Z$ is in $Z$, the product of two sets in $P$ is in $P$, and the product of a set in $Z$ and a set in $P$ is in $Z$. Thus 
$Z+P$ is closed under the product operation. The proof of part (2) remains valid if we replace the assumptions $\alpha, \beta \in Z$ by $\alpha \in Z, \beta \in F$. Let $\alpha \in Z+P$ and $\beta \in F$; then either $\alpha \in Z$ hence $\alpha+\beta \in Z+P$, or $\alpha \in P$ hence $\alpha+\beta \in P$. Thus $\alpha+\beta \in Z+P$. This completes the proof.

THEOREM 1.6. (1) The product of two hypersimple sets is hypersimple.

(2) The sum of two hypersimple sets is either hypersimple or belongs to $P$.

(3) There exist two hypersimple sets whose sum equals $\epsilon$.

(4) $Z_{0}+P$ is a dual ideal in the lattice $F$.

Proof. (1) Let $\alpha, \beta \in Z_{0}$ and let $\left\{\gamma_{n}\right\}$ be a discrete array. Suppose $\rho$ is the set of all non-negative integers $n$ such that $\beta \supset \gamma_{n}$, then $\rho$ is infinite by Theorem 1.4. Let $\Gamma_{i}$ be the act of comparing the first $i$ elements of $\beta$ with the first $i$ rows of $\left\{\gamma_{n}\right\}$, then we can effectively generate $\rho$ by performing the acts $\Gamma_{0}, \Gamma_{1}, \cdots$. Thus $\rho$ is r.e.; suppose $r(n)$ is a 1-1 recursive function ranging over $\rho$ and suppose $\delta_{n}={ }_{d f} \gamma_{r(n)}$. Then $\left\{\delta_{n}\right\}$ is a discrete array which is a subarray of $\left\{\gamma_{n}\right\}$. Since $\beta \supset \delta_{n}$ for all values of $n$ and $\alpha \supset \delta_{n}$ for infinitely many values of $n$, it follows that $\alpha \beta \supset \gamma_{n}$ for infinitely many values of $n$. We conclude $\alpha \beta \in Z_{0}+P$. Clearly $\alpha \beta \notin P$ because $\alpha \notin P$. Thus $\alpha \beta \in Z_{0}$.

(2) Let $\alpha, \beta \in Z_{0}$. Either $\alpha+\beta \in P$ or $\alpha+\beta \notin P$. In the latter case $\alpha+\beta \in Z_{0}$, since $\alpha \subset \alpha+\beta$ and $\alpha \in Z_{0}$.

(3) Using the fact that $Z_{0}$ is recursively closed we can prove this part similarly to the third part of Theorem 1.5.

(4) The proof of part (2) remains valid if we replace the assumptions $\alpha, \beta \in Z_{0}$ by $\alpha \in Z_{0}, \beta \in F$. We can now prove this part in the same way as the fourth part of Theorem 1.5.

\section{Note 2. A MESOIC SET}

1. Preliminaries. Let $\Phi(n, x)$ be the partial recursive function discussed by Kleene ${ }^{2}$ which generates all partial recursive functions of one variable. We shall denote this function by $g_{n}(x)$. Following Rice we use $g_{n}(x)$ to characterize r.e. sets. Let $\omega_{n}$ denote the range of $g_{n}(x)$, then $\left\{\omega_{n}\right\}$ is a sequence of r.e. sets in which every r.e. set occurs at least once. The set $\alpha$ is called productive if there exists a partial recursive function $p(n)$ such that $\omega_{n} \subset \alpha$ implies: (1) $p(n)$ is defined, (2) $p(n) \in \alpha-\omega_{n}$. Every such function $p(n)$ is called a productive function of $\alpha$. Let Dom $\alpha$ denote the set of all $n$ such that $\omega_{n} \subset \alpha$. The subset

2 Recursive predicates and quantifiers, Trans. Amer. Math. Soc. vol. 53 (1943) pp. 41-73.

3 Classes of recursively enumerable sets and their decision problems, Trans. Amer. Math. Soc. vol. 74 (1953) pp. 358-366. 
$\pi$ of $\alpha$ is called a productive center of the productive set $\alpha$, if $\pi$ $=p(\operatorname{Dom} \alpha)$ for some productive function $p(n)$ of $\alpha$. The set $\alpha$ is called productive in the sense of Post (abbreviated: $P$-productive), if at least one of its productive functions is recursive. The set $\alpha$ is called creative (or $P$-creative), if $\alpha$ is r.e. and $\alpha^{\prime}$ productive (respectively $P$-productive). Clearly, every $P$-productive set is productive and every $P$-creative set is creative.

The class of all creative sets is denoted by $H$. The question arises whether $H$ and $Z$ exhaust $F-E$. It is the purpose of this note to answer this question in the negative. The set $\alpha$ is called medial if it is not r.e. and neither immune nor productive. The set $\alpha$ is called mesoic if $\alpha$ is r.e. and $\alpha^{\prime}$ medial, i.e., if $\alpha \in(F-E)-(Z+H)$.

We recall that $\alpha$ is many-one reducible to $\beta$ [denoted by: $\alpha(m-1) \operatorname{red} \beta]$, if there exists a recursive function which maps $\alpha$ into $\beta$ and $\alpha^{\prime}$ into $\beta^{\prime} ; \alpha$ is one-one reducible to $\beta$ [denoted by : $\alpha$ (1-1)red $\beta$ ], if there exists a 1-1 recursive function which maps $\alpha$ into $\beta$ and $\alpha^{\prime}$ into $\beta^{\prime}$. The degree of unsolvability of $\alpha$ relative to $(m-1)$ reducibility [or (1-1) reducibility] is denoted by $\Delta(\alpha)$ [respectively by: $d(\alpha)$ ]. If $\alpha(m-1)$ red $\beta$ we write $\Delta(\alpha) \leqq \Delta(\beta)$; if $\alpha(m-1)$ red $\beta$ is true, but $\beta(m-1)$ red $\alpha$ is false, we write $\Delta(\alpha)<\Delta(\beta)$. Similarly $d(\alpha) \leqq d(\beta)$ and $d(\alpha)<d(\beta)$ are defined.

The following theorems ${ }^{4}$ will be used.

A. If $f(x)$ is a partial recursive function defined for at least one value of $x$, we can effectively find a recursive function whose range is the same as that of $f(x)$.

B. The set $\alpha$ is productive if and only if $\alpha \neq 0$ and there exists a partial recursive function $p(n)$ such that $\omega_{n} \neq 0$ and $\omega_{n} \subset \alpha$ imply: (1) $p(n)$ is defined, (2) $p(n) \in \alpha-\omega_{n}$.

REMARK. Theorem $B$ remains valid if we replace "productive" by "P-productive."

TheOREM 2.1. If $\alpha(m-1)$ red $\beta$ and $\alpha$ is productive, then $\beta$ is productive.

Proof. There exists a recursive function which maps $\alpha$ into $\beta$ and $\alpha^{\prime}$ into $\beta^{\prime}$, say $f(n)$. Suppose $\omega_{n} \neq 0$. By Theorem A we can now from the function $g_{n}(x)$ effectively find a partial recursive function ranging over $\omega_{n}$, say $d(x)$. By comparing $f(0), \cdots, f(k)$ with $d(0), \cdots, d(k)$ for $k=0,1, \cdots$, we can effectively find a recursive function ranging over $f^{-1}\left(\omega_{n}\right)$. Thus $f^{-1}\left(\omega_{n}\right)$ is r.e. If $\omega_{n} \subset \beta$, we know $f^{-1}\left(\omega_{n}\right) \subset \alpha$ and by

\footnotetext{
- These theorems are discussed in the author's paper Productive sets, not yet published.
} 
the productivity of $\alpha$ we can effectively find an element $a \in \alpha-f^{-1}\left(\omega_{n}\right)$. Then $f(a) \in \beta-\omega_{n}$. We conclude that $\beta$ is productive.

REMARK. It easily follows from this proof that the theorem remains valid if we replace "productive" by " $P$-productive" at both of its occurrences.

TheOREM 2.2. If $\alpha(m-1)$ red $\beta, \alpha$ is creative and $\beta$ is r.e., then $\beta$ is creative.

Proof. $\alpha^{\prime}(m-1)$ red $\beta^{\prime}$ since $\alpha(m-1)$ red $\beta$. But $\alpha^{\prime}$ is productive, hence $\beta^{\prime}$ is productive. We conclude that $\beta$ is creative.

REMARK. This theorem remains valid if we replace "creative" by "P-creative" at both of its occurrences.

Post proved [RES p. 295] that the complete set $\kappa$ is $P$-creative by showing that $\kappa$ is r.e. and $\kappa^{\prime} P$-productive. The theorem mentioned in the last remark enables us to give a different proof of this fact. Clearly $\alpha$ is r.e. Let $\alpha={ }_{d f} \widehat{n}\left[n \in \omega_{n}\right]$; from Post's proof [RES pp. 291, 292] that $\alpha \in F-E$ it follows immediately that $\alpha^{\prime}$ is $P$-productive with the identity function as one of its productive functions. Thus $\alpha$ is $P$-creative. Observe that $\alpha(1-1)$ red $\kappa$, since every r.e. set is one-one reducible to the complete set [RES p. 297]. Then $\kappa$ is $P$-creative because $\kappa$ is r.e. and there exists a $P$-creative set (namely $\alpha$ ) which is many-one reducible to $\kappa$.

\section{The main result.}

THEOREM 2.3. There exists a set $\nu$ such that:

(1) $\nu$ is mesoic.

(2) $d(\zeta)<d(\nu)<d(\kappa)$.

(3) $\Delta(\zeta)=\Delta(\nu)<\Delta(\kappa)$.

Proof. (1) Let $\alpha$ be the set of all even non-negative integers, $\beta$ the set of all odd non-negative integers, and $z(n)$ a 1-1 recursive function ranging over $\zeta$. Suppose $\alpha_{1}$ is the range of the function $2 \cdot z(n)$ and $\alpha_{2}={ }_{d f} \alpha-\alpha_{1}$. Then $\epsilon=\alpha_{1}+\alpha_{2}+\beta$, where $\alpha_{1}, \alpha_{2}, \beta$ are mutually disjoint. We shall prove that $\alpha_{1}$ is mesoic. The fact that $\zeta^{\prime}$ is immune implies that $\alpha_{2}$ is immune, since the mapping $n \rightarrow 2 n$ maps $\zeta^{\prime}$ recursively and 1-1 on $\alpha_{2}$. Clearly, $\alpha_{1}$ is r.e. To complete the proof it is now sufficient to prove that $\alpha_{1}^{\prime}$ is medial. Observe that $\alpha_{1}^{\prime}=\alpha_{2}+\beta$. First of all, $\alpha_{2}+\beta$ is not r.e., for if it were $\alpha_{2}=\left(\alpha_{2}+\beta\right) \beta^{\prime}$ would be r.e., while we know that $\alpha_{2}$ is immune. Secondly $\alpha_{2}+\beta$ is not immune because it includes the infinite r.e. set $\beta$. Now suppose $\alpha_{2}+\beta$ were productive. Then we could effectively find an element $c_{0} \in\left(\alpha_{2}+\beta\right)-\beta$ $=\alpha_{2}$, an element $c_{1} \in\left(\alpha_{2}+\beta\right)-\left(\beta+\left\{c_{0}\right\}\right)=\alpha_{2}-\left\{c_{0}\right\}$, etc. Then the 
immune set $\alpha_{2}$ would include the infinite r.e. set $\left\{c_{0}, c_{1}, \cdots\right\}$, which is impossible. Thus $\alpha_{1}^{\prime}$ is medial and $\alpha_{1}$ mesoic. From now on we shall denote the set $\alpha_{1}$ by $\nu$.

(2) $d(\nu) \leqq d(\kappa)$, since every r.e. set is $1-1$ reducible to $\kappa$. But $d(\kappa) \leqq d(\nu)$ is impossible because of Theorem 2.2 and the fact that $\nu$ is not creative. Thus $d(\nu)<d(\kappa)$. It follows from the definition of $\nu$ that the 1-1 recursive function $f(n)=2 n$ maps $\zeta$ on $\nu$ and $\zeta^{\prime}$ into $\nu^{\prime}$. Hence $d(\zeta) \leqq d(\nu)$. But $d(\nu) \leqq d(\zeta)$ is impossible, since $\nu^{\prime}$ does include an infinite r.e. set (namely $\beta$ ), while $\zeta^{\prime}$ does not. Thus $d(\zeta)<d(\nu)$.

(3) $d(\zeta)<d(\nu)$ implies $\Delta(\zeta) \leqq \Delta(\nu)$. Recall the definition of $\nu$. Suppose $b$ is any element of $\zeta^{\prime}$. Let $f(n)={ }_{d f} n / 2$ if $n$ is even (i.e., for $n \in \alpha$ ) and $f(n)={ }_{d f} b$, if $n$ is odd (i.e., for $n \in \beta$ ). Then $f(n)$ is a recursive function which maps $\alpha_{1}$ on $\zeta, \alpha_{2}$ on $\zeta^{\prime}$, and $\beta$ on $\{b\}$; hence $f(n)$ inaps $\nu$ on $\zeta$ and $\nu^{\prime}$ into $\zeta^{\prime}$. It follows that $\Delta(\nu) \leqq \Delta(\zeta)$. Consequently $\Delta(\zeta)$. $=\Delta(\nu) . d(\nu)<d(\kappa)$ implies $\Delta(\nu) \leqq \Delta(\kappa)$. But $\Delta(\kappa) \leqq \Delta(\nu)$ is impossible because of Theorem 2.2 and the fact that $\nu$ is not creative. Thus $\Delta(\nu)<\Delta(\kappa)$. This completes the proof.

University of Chicago 